\title{
Systematic behavior of electro-optic chromophore photostability
}

\author{
Adriana Galvan-Gonzalez, Michael Canva, * and George I. Stegeman \\ School of Optics and Center for Research and Education in Optics and Lasers, University of Central Florida, \\ 4000 Central Florida Boulevard, Orlando, Florida 32816-2700 \\ Robert Twieg \\ Department of Chemistry and Liquid Crystal Institute, Kent State University, Kent, Ohio 44242
}

Kwok Pong Chan

Molecular OptoElectronics Corporation, 877 25th Street, Watervliet, New York 12189-1903

Tony C. Kowalczyk, Xuan Q. Zhang, and Hilary S. Lackritz

Gemfire Corporation, Suite 600, 2471 East Bayshore Road, Palo Alto, California 94303

Seth Marder and Sankaran Thayumanavan

Department of Chemistry, University of Arizona, Tucson, Arizona 85721

Received October 8, 1999

\begin{abstract}
The wavelength dependence of the one-photon absorption-induced photodegradation rate has been measured from the visible to the near IR for a variety of electro-optic chromophore-doped polymers. Systematic behavior is identified. The lifetime of the electro-optic activity is found to increase exponentially over 4-6 orders of magnitude for wavelengths ranging from peak of absorption, typically in the visible, to $\sim 1000 \mathrm{~nm}$. Many popular chromophores developed for electro-optics over the past 10 years are compared. (๐) 2000 Optical Society of America

OCIS codes: $190.0190,250.2080$
\end{abstract}

During the past decade certain aspects of polymers that contain chromophores have been investigated intensively, and the performance of polymers required for a variety of photonics applications has improved steadily. ${ }^{1,2}$ However, to use these highly efficient materials one must address their stability. The temporal stability of the chromophore orientation necessary for electro-optic activity to be sustained has been investigated intensively. ${ }^{3}$ Photostability has been less well explored, and the reported studies have often been made over limited wavelength ranges, usually only in the visible or the infrared..$^{4-9}$

The same chromophores being studied for electrooptics are also used near their absorption maxima for holography, data storage, writing waveguides and gratings, etc. based on low-power, optically induced changes in their refractive indices. ${ }^{10}$ But electrooptic devices operating in the wavelength window $820-1550 \mathrm{~nm}$ are expected to last years without significant degradation. Therefore it is important to investigate the photostability of chromophore-doped material at device operating wavelengths. Here we address a number of key questions: (1) What is the relationship between the photostability of these chromophores and photon energy (wavelength)? (2) Are current materials sufficiently photostable for applications at 820 and $1300 \mathrm{~nm}$ ? (3) Are there molecular structures that should be avoided? Specifically, we report measurements of the relationship between photostability and photon energy for typical electro-optic active chromophores from 450 to $1300 \mathrm{~nm}$.

0146-9592/00/050332-03\$15.00/0
The photodegradation processes can be summarized in the following way: The absorption of light results in raising the chromophore to an excited state from which the molecule has a certain probability, quantum efficiency $B^{-1}$, of undergoing an irreversible chemical transformation to a new species that is effectively not electro-optically active. The temporal evolution under irradiation can easily be modeled. ${ }^{11}$ This schematic two-step process results in a cumulative loss of the material's nonlinearity with time as progressively more of the active chromophores are removed. Our recent studies of 4-dimethylamino-4'-nitrostilbene (DANS) and 4-N-ethyl-N-(2-hydroxyethly)amino]-4'nitroazobenzene] (DR1) have shown that the dominant degradation mechanism is photo-oxidation. ${ }^{12,13}$

The local rate of creation of the electro-optic inactive photoproduct $N_{2}$ is expressed as $\partial N_{2} / \partial t=$ $B^{-1}(\lambda) \partial N_{E} / \partial t=[\sigma(\nu) / B(\nu)] N_{1}(t) n(t)$, where $N_{E}$ is the number of electro-optic chromophores in their excited state and $N_{1}(t), n(t)$, and $\sigma(\nu)$ are, respectively, the local electro-optic active chromophore concentration, the photon flux, and the molecular absorptivity. Thus, the key parameter that describes the decay time of the population of the active species is $\tau=B / \sigma n$, where $B / \sigma$ is the photostability figure of merit. The larger $B / \sigma$, the more stable the doped polymer material.

We investigated more than 40 chromophores, and representative examples of their chemical structures are listed in Table 1. Except for the side-chain polymers (SCP's) DANS and DR1, all chromophores were studied as guest-host material systems. Each 
material was deposited as a film onto glass substrates $\sim 2 \mu \mathrm{m}$ thick by use of spin coating. Photodegradation was induced by illumination with strong pump beams from 430 to $1300 \mathrm{~nm}$. For charge-transfer chromophores the macroscopic electro-optic activity is proportional to the quantity of active chromophores present and hence to the strength of the chargetransfer absorption feature. We determined the concentration of chromophores by monitoring with a 543.5-nm probe beam (He-Ne laser, weak enough not to induce photodegradation) the temporal evolution of the transmission change. The probe beam's transmission initially increases with time (i.e., the integrated flux), and the initial slope of the transmission change directly yields the ratio $B / \sigma$. We verified that the evolution of the degradation curves depended solely on the integrated flux at different average pump power levels.

Representative results for $(B / \sigma)(\nu)$ indicative of the trends measured in all the materials are shown in Fig. 1. Four clear trends emerge: (1) an increase in $B / \sigma$ (and hence in chromophore lifetime) over orders of magnitude with decreasing photon energy $h \nu$; (2) a distinct linear dependence of $\log (B / \sigma)$ on $h \nu$ a few tens of percent above the absorption maximum $h \nu_{\max }$ (associated with the ground state to charge-transfer state transition) to $1.25 \mathrm{eV}$; (3) a relative decrease in $B / \sigma$ near $1 \mathrm{eV}(1320 \mathrm{~nm})$, possibly owing to singlet oxygen as identified previously; ${ }^{4}$ and (4) a relatively constant $B / \sigma$ for $h \nu>h \nu_{\max }$.

We have precise absorption measurements over the full wavelength range only for the stilbene SCP DANS (weight fraction, $43 \%$ ). ${ }^{14}$ The inverse absorption coefficient $\alpha^{-1}(\sigma \propto \alpha)$ exhibits (Fig. 2) an approximately logarithmic behavior relative to photon energy over the range $550-1000 \mathrm{~nm}$, the same spectral region in which $\log (B / \sigma)$ is linear in photon energy. There $\sigma(\nu)$ dominates the wavelength dependence of $B / \sigma$. The same $\log (B / \sigma)$ dependence was found in the other chromophores studied. The plateau in $B / \sigma$ near $h \nu_{\max }$ is a consequence of $\sigma(\nu)$ varying by only a factor of 2 over the full half-width $(100-200 \mathrm{~nm})$ of the absorption spectrum. Note that $B / \sigma$ typically rises with further increase in $\nu$ on the high-energy side of $\nu_{\max }$, as expected; e.g., chromophore 4 in Table 1 . The situation in DANS for $\nu>\nu_{\max }$ is more complex because of the presence of a second, higher-energy charge-transfer state with a different $B .^{12}$

Figure 3 shows the dependence of $B$ on $h \nu$ for a few cases. Typically $B$ is independent of wavelength. However, in the SCP DANS there are two distinct values of $B$ that we associate with photodegradation of two distinct charge-transfer states, located at 4.17 and $2.90 \mathrm{eV} .^{11} \quad B$ varies by 4 orders of magnitude from chromophore to chromophore. The larger $B$, the more stable the chromophore.

The log-linear behavior observed for $B / \sigma$ implies that

$$
B / \sigma=D_{0} \exp \left(-h \nu / E_{0}\right),
$$

where $D_{0}$ and $E_{0}$ are constants. This characteristic behavior allows $B / \sigma$ to be estimated over the relevant wavelength range from measurements of $B / \sigma$ at just two different values of $\nu$. In a plot of $D_{0}$ versus $E_{0}$ (Fig. 4), there is a small spread in $E_{0}$ clustered about $0.085 \mathrm{eV}$, in contrast to $D_{0}$, which varies over 7 orders of magnitude, which implies that even one measurement at one $\nu$ permits a good estimate over the whole visible-near-IR spectral range. As discussed above, $E_{0}$ depends on the absorption spectrum on the lowenergy side. Our overall results indicate only a weak

Table 1. Molecular Structures Studied at CREOL of Chromophores as Guests in Poly(Methyl Methacrylate) Host B Values in the Plateau Region, Location of $\lambda_{\text {max }}$, and Symbols Used for Each in Figs. 1-4

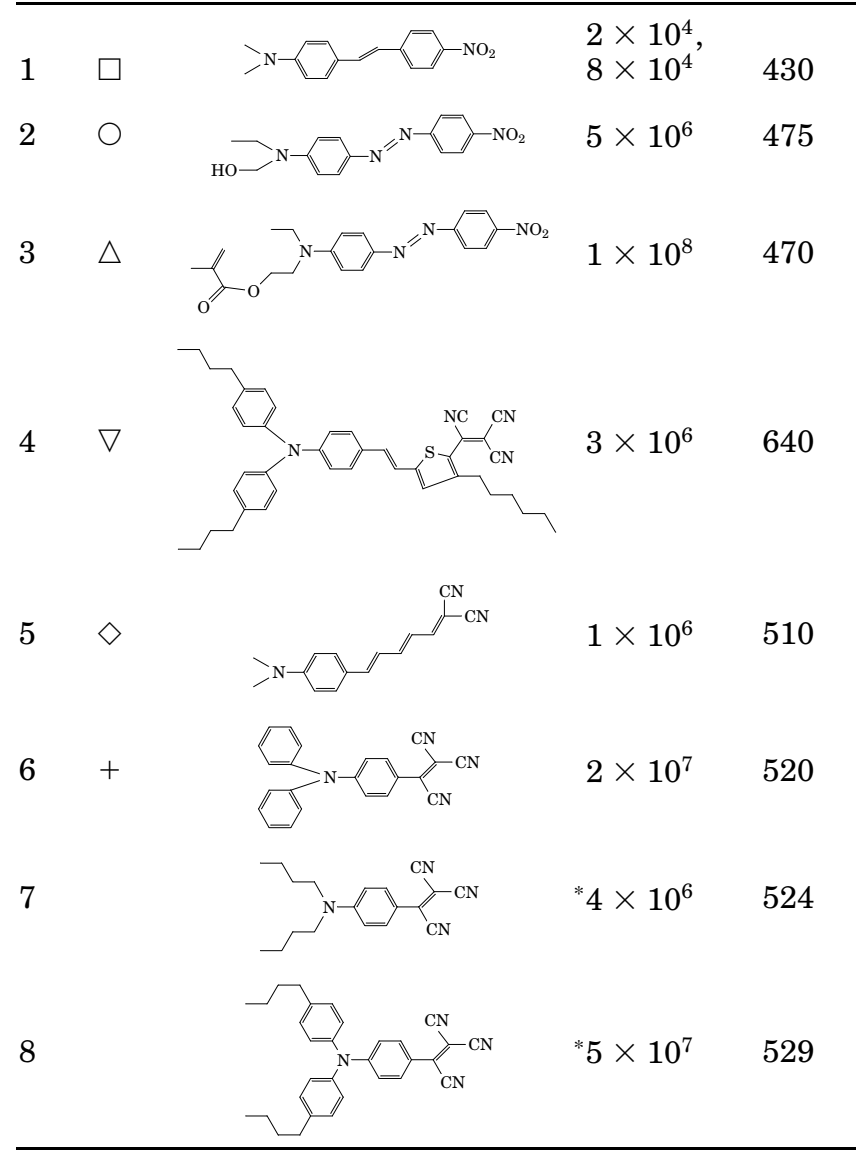

${ }^{a}$ An asterisk identifies a value taken at Gemfire at $5 \mathrm{~nm}$ only.

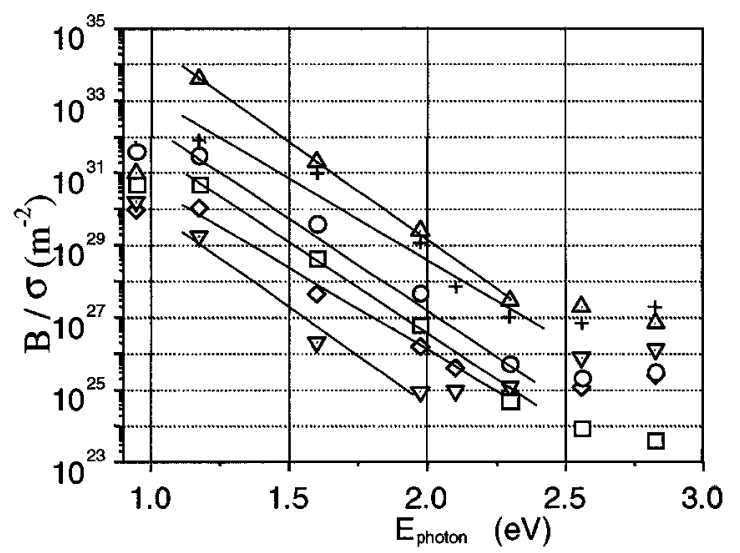

Fig. 1. $\log (B / \sigma)$ versus photon energy, $E_{\text {photon }}=h \nu_{\text {pump }}$, for a representative range of materials listed in Table 1. 


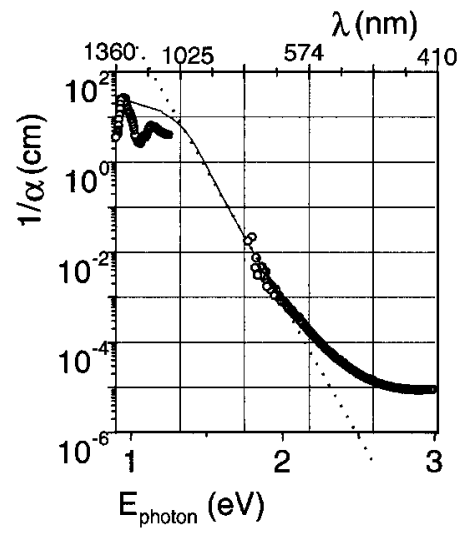

Fig. 2. $\alpha^{-1}$ of DANS, measured as film transmission in the visible and as waveguide propagation loss in the IR. The experimental data are fitted by use of a Voigt profile. Absorption for $\lambda>1000 \mathrm{~nm}$ shows maxima owing to vibrational overtones.

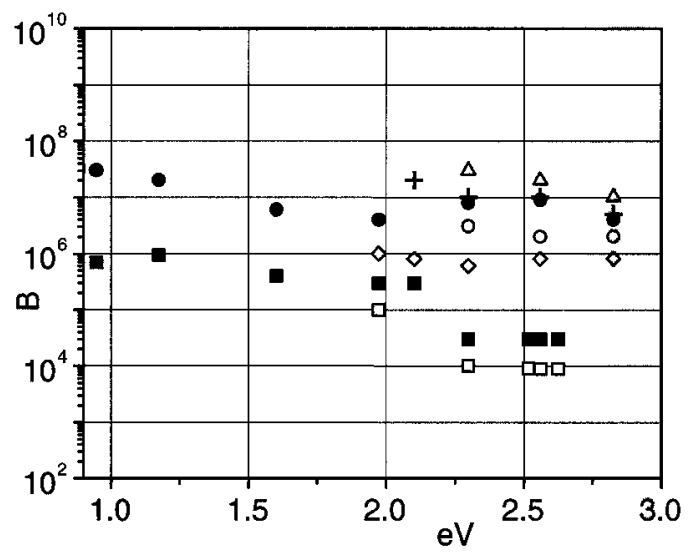

Fig. 3. Photostability parameter $B$ as a function of excitation photon energy, $E_{\text {photon }}=h \nu_{\text {pump }}$, for the SCP's DANS (ם) and DR1 (O) and the guest-host cases indicated by the various types of points.

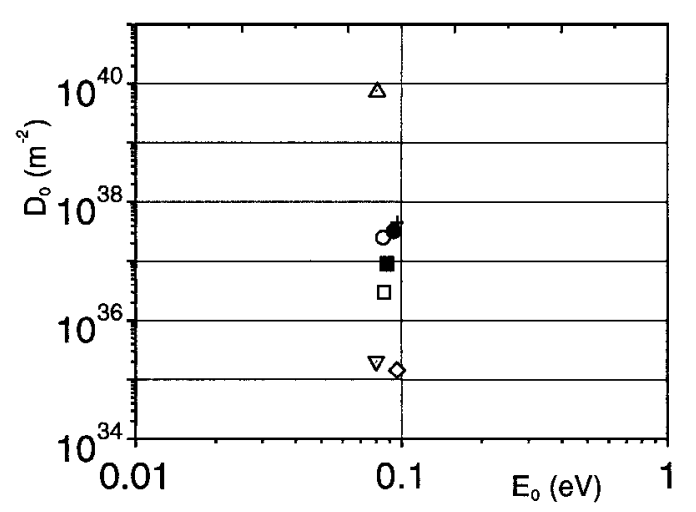

Fig. 4. Values of $D_{0}$ and $E_{0}$ for the polymers listed for Fig. 3.

dependence of $E_{0}$ on the details of the chromophore structure, the host matrix, and whether the polymer is side chain or guest-host. In contrast, the prefactor $D_{0}$ depends strongly on the details of the molecular structure, the host, etc.
A prime application of such polymers is to electrooptic channel waveguide devices expected to operate for 10 years under continuous illumination. ${ }^{1}$ Assuming a waveguide area of $10 \mu \mathrm{m}^{2}$ and a $1-\mathrm{mW}$ power level, $B / \sigma \geq 10^{35}$ is needed to reach this lifetime. This requires $B \geq 10^{9}$, already reached for solid-state dye laser molecules. ${ }^{15}$ Some of the current materials studied come within 1 order of magnitude of this value.

This research was supported by the National Science Foundation (NSF) at the University of Central Florida (CREOL) and the University of Arizona and by the Ballistic Missile Defense Organization at Gemfire Corporation. CREOL and the Université d'Orsay also acknowledge bilateral U.S./French NSF/Centre National de la Recherche Scientifique collaboration support. G. Stegeman's e-mail address is george@creol.ucf.edu.

*Permanent address, Laboratoire Charles Fabry de l'Institut d'Optique, Centre National de la Recherche Scientifique, Unité Mixte de Recherche 8501, Université d'Orsay-Paris XI, 91403 Orsay Cedex, France.

\section{References}

1. For example, Y. Shi, W. Wang, J. H. Bechtel, A. Chen, S. Garner, S. Kalluri, W. H. Steier, D. Chen, H. R. Fetterman, L. R. Dalton, and L. Yu, IEEE J. Sel. Top. Quantum Electron. 2, 289 (1996).

2. For example, A. Grunnet-Jepsen, C. L. Thompson, R. J. Twieg, and W. E. Moerner, Appl. Phys. Lett. 70, 1515 (1997).

3. For example, D. H. Choi, J. H. Park, N. Kim, and S.-D. Lee, Chem. Mater. 10, 705 (1998).

4. M. A. Mortazavi, H. N. Yoon, and C. C. Teng, J. Appl. Phys. 74, 4871 (1993); M. A. Mortazavi, H. N. Yoon, and I. McCulloch, Polym. Prepr. Am. Chem. Soc. Div. Polym. Chem. 35, 198 (1994); R. A. Norwood, D. R. Holcomb, and F. F. So, J. Nonlin. Opt. 6, 193 (1993).

5. M. Cha, W. E. Torruellas, G. I. Stegeman, and W. H. G. Horsthuis, Appl. Phys. Lett. 65, 2648 (1994).

6. Y. Shi, W. Wang, W. Lin, D. J. Olson, and J. H. Bechtel, Appl. Phys. Lett. 70, 1342 (1997).

7. Q. Zhang, M. Canva, and G. I. Stegeman, Appl. Phys. Lett. 73, 912 (1998)

8. Ph. Pretre, E. Sidlick, A. Knoesen, D. J. Dyer, and R. J. Twieg, ACS Symp. Ser. 695, 328 (1996).

9. T. C. Kowalczyk, R. J. Twieg, and H. S. Lackritz, presented at the Fourth International Conference on Organic Nonlinear Optics, October 12-14, 1998, Chitose, Japan.

10. For example, T. Todorov, L. Nikolova, and N. Tomova, Appl. Opt. 23, 4309 (1984); Y. Shi and W. H. Steier, Appl. Phys. Lett. 59, 2935 (1991); M. Ivanov, T. Todorov, L. Nikolova, N. Tomova, and V. Dragostinova, Appl. Phys. Lett. 66, 2174 (1995).

11. A. Dubois, M. Canva, A. Brun, F. Chaput, and J.-P. Boilot, Appl. Opt. 35, 3193 (1996).

12. A. Galvan-Gonzalez, M. Canva, and G. I. Stegeman, Appl. Phys. Lett. 75, 3306 (1999).

13. A. Galvan-Gonzalez, M. Canva, G. I. Stegeman, R. Twieg, C. Kowalczyk, H. S. Lackritz, and K. P. Pong, Opt. Lett. 24, 1741 (1999).

14. A. Otomo, M. Jäger, G. Stegeman, M. Flipse, and M. Diemeer, Appl. Phys. Lett. 69, 1991 (1996).

15. A. Dubois, M. Canva, A. Brun, F. Chaput, and J.-P. Boilot, Synth. Metals 81, 305 (1996). 